\title{
Application of First-Order Differential Equation to Heat Convection in Fluid
}

\author{
Zahidullah Rehan \\ Department Mathematics and Physics, Sayed Jamaluddin Afghani University, Asadabad, Afghanistan \\ Email: rehanzahidullah@gmail.com
}

How to cite this paper: Rehan, Z. (2020) Application of First-Order Differential Equation to Heat Convection in Fluid. Journal of Applied Mathematics and Physics, 8, 1456-1462.

https://doi.org/10.4236/jamp.2020.88111

Received: May 27, 2020

Accepted: August 3, 2020

Published: August 6, 2020

Copyright $\odot 2020$ by author(s) and Scientific Research Publishing Inc. This work is licensed under the Creative Commons Attribution International License (CC BY 4.0).

http://creativecommons.org/licenses/by/4.0/

(c) (i) Open Access

\begin{abstract}
Differential equation is very important in science and engineering, because it required the description of some measurable quantities (position, temperature, population, concentration, electrical current, etc.) in mathematical form of ordinary differential equations (ODEs). In this research, we determine heat transferred by convection in fluid problems by first-order ordinary differential equations. So in this research work first we discuss the solution of ordinary homogeneous and non-homogeneous differential equation and then apply the solution of first-order ODEs to heat transferring particularly in heat convection in fluid.
\end{abstract}

\section{Keywords}

Differential Equation, Heat Transfer Analysis, Heat Convection in Fluid, Newton Cooling Law

\section{Introduction}

There are a lot of differential equations which become from different application of mathematics. Differential equations contain only first-order derivatives known as first-order differential equation. These equations have many applications in daily life such as used in engineering, physics, biology etc. In the year 1675 Gottfried Wilhelm Von Leibniz studied the integration $\int x \mathrm{~d} x=\frac{1}{2} x^{2}$. Then he knows the ODEs for the first time [1].

In more general terms, a deferential equation is simply an equation involving unknown function and its derivatives. To be more technical, differential equation is a "mathematical equation for an unknown function of one or several variables that relate the values of the function itself and its derivatives of various orders" to a particular phenomenon. Differential equations generally fall into 
two categories, ordinary differential equation and partial differentials equations, the distinction being that ODEs involve unknown functions of one independent variable while PDEs involve unknown functions of more than one independent variable. In this paper we will focus on first-order ordinary differential equation [2].

Karthikeyan and Srinivasan studied the first-order homogeneous and nonhomogeneous differential equation and discovered that in the area of heat transferring in the solid. These equations have a lot of application in this area [3].

The water found around the word has temperature range between $\left(0^{\circ} \mathrm{C}\right.$ for freshwater and $-1.9^{\circ} \mathrm{C}$ for sea water) and $\left(100^{\circ} \mathrm{C}\right.$ for fresh water and $102^{\circ} \mathrm{C}$ for sea water), where the freezing point is $0^{\circ} \mathrm{C}$ for fresh water and $-1.9^{\circ} \mathrm{C}$ for sea water and boiling point is $\left(100^{\circ} \mathrm{C}\right.$ for fresh water and $102^{\circ} \mathrm{C}$ for sea water). The water which is in the ocean is known as free water and its temperature is at the low end of the above range and at average temperature approximately one $1^{\circ} \mathrm{C}$ this became frozen. Warm water is found in some location on the point. A little amount of earth water, around $0.5 \%$ with typical temperature is contained under the ground. Similarly to the annual air temperature all lake and river contain only $\left(0.01 \%\right.$ of the earth free water) and freezing point is $0^{\circ} \mathrm{C}$ and boiling point is $40^{\circ} \mathrm{C}$ respectively. The temperature of shallow lakes and river can reach $40^{\circ} \mathrm{C}$ in one climate. Thus the maximum temperature of most lake and stream are somewhat less than this extreme [4].

Hassan A. and Zakari Y. studied the first-order ordinary differential equation and discovered that it has many applications in temperature problems which lead to use Newton's cooling law and obtain the solution of some practical problems that arise from the $1^{\text {st }}$ order ODEs [5].

On the conference on mathematical modeling in continuum mechanics and inverse problems of finding the solutions of differential equations together with one or several coefficients of the equations or the coefficients and an unknown external force, the same problem can be used for the elliptic, parabolic and hyperbolic equation, the equation with multiple characteristic and solvable kind of equation [6].

In this research article we divided the first-order differential equation in two parts, the equation in which the coefficient is constant known as homogeneous differential equation and the equation in which the coefficient is function or polynomial is known as the non-homogeneous differential equation. The aim and scope of this paper is to know the type of first-order ordinary differential equation, which one is homogeneous and which one is non-homogeneous differential equation. Also in this paper we will discuss the solution method of first-order linear homogeneous and non-homogeneous differential equation and apply it to solve the heat transferring problems especially heat convection in fluid problems that required the use of Newton's cooling law.

\section{Differential Equation and Its Solution}

An equation which has one or more derivatives of differentials is known as dif- 
ferential equation. Differential equation that depend only on a single variable known as ordinary differential equation but equation which depend on more than one variable known as partial differential equation [5].

$$
\begin{aligned}
& y^{\prime}+h(x) y=g(x) . \\
& x z \frac{\partial z}{\partial x}+y z \frac{\partial z}{\partial y}=-3 x y .
\end{aligned}
$$

Equation (1) is first-order differential equation because they involve only first derivative of the function and no higher derivatives.

\section{Solution of First-Order ODEs}

Here we discuss the solution of linear differential equation which divides in to two type one is of the form $y^{\prime}+h(x) y=0$ is known as homogeneous linear differential equation and its solution is $y=\frac{c}{u(x)}$ where is the constant of integration to be determined and $u(x)$ is known as the integrating factor can be obtain from the equation [7].

$$
u(x)=\mathrm{e}^{\int-h(x) \mathrm{d} x} .
$$

If the first-order differential equation has the form $y^{\prime}+h(x) y=g(x)$ is called non homogeneous differential equation. The solution of this equation is similar with a little difference of the homogeneous differential equation $y^{\prime}+h(x) y=0$

$$
y=\frac{1}{u(x)}\left(\int u(x) \cdot g(x) \mathrm{d} x+a\right) .
$$

where the function $u(x)$ can be obtain from Equation (3) [5].

\section{Example 1.}

Obtain the solution of the initial value problem.

$$
y^{\prime}-y \cdot \tan x=\sin x \text { where } y(0)=1 .
$$

\section{Solution.}

First we calculate integrating factor $u(x)$.

$$
u(x)=\mathrm{e}^{\int-h(x) \mathrm{d} x}=\mathrm{e}^{-\int \tan x \mathrm{~d} x} .
$$

Here

$$
\int \tan (x) \mathrm{d} x=\int \frac{\sin (x)}{\cos (x)} \mathrm{d} x=\int \frac{-\mathrm{d}(\cos (x))}{\cos (x)}=-\int \frac{\mathrm{d}(\cos (x))}{\cos (x)}=-\ln |\cos (x)| .
$$

Hence $u(x)$ is given by

$$
u(x)=\mathrm{e}^{\ln |\cos x|}=|\cos x| .
$$

We can take the function $u(x)=\cos x$ as the integrating factor make sour that the L.H.S of the equation is the derivative of the product $y(x) u(x)$.

Thus the general solution of the equation is

$$
y(x)=\frac{1}{u(x)}\left[\int u(x) \sin x \mathrm{~d} x+c\right]=\frac{1}{\cos x}\left[\int \cos x \cdot \sin x \mathrm{~d} x+c\right]=\frac{c}{\cos x}-\frac{\cos 2 x}{4 \cos x} .
$$


Now we obtain the value of the constant $c$ by putting $y(0)=1$ in the above equation, thus we get

$$
y(0)=\frac{c}{\cos 0}-\frac{\cos 0}{4 \cos 0} \Rightarrow 1=c-\frac{1}{4} \Rightarrow c=\frac{5}{4} .
$$

Hence the solution of the given problem is

$$
y(x)=\frac{5}{4 \cos x}-\frac{\cos 2 x}{4 \cos x}=\frac{5-\cos 2 x}{4 \cos x}[1] .
$$

\section{Application of the First of Order Differential Equation to the Heat Convection in Fluid}

\subsection{Heat Transferring}

The transmission of thermal energy in the physical system depend in on the temperature and pressure, by dissipating heat is describes by heat transforming, heat generally transforming by conduction or diffusion, convection and radiation. Heat is always transmitted by one of the following three modes [3].

- Heat transferring by conduction in solid;

- Transferring of heat by convection in fluid;

- Transferring of heat by radiation in space.

In "Figure 1" we see that the solids have contact with fluid at different temperature.

Thus, heat flows in the solid by conduction which can be determined by Fourier law in the solid heat always flow from high to low temperature. But in fluid heat flowing by convection which can be calculated by Newton's cooling law.

\subsection{Mathematical Model of Heat Transformation in Fluid}

Since heat flow in the fluid by convection "as shown in Figure 2" so we can determined it the following equation which known as the Newton's cooling law.

$$
q \alpha\left(T_{a}-T_{b}\right)=h\left(T_{a}-T_{b}\right) .
$$

Equation (5) show that heat flows from $T_{a}$ to $T_{b}$ with $T_{a}>T_{b}$ which express the heat flux between two point $\mathrm{A}$ and $\mathrm{B}$ and $\mathrm{h}$ is heat transfer coefficient. Thus
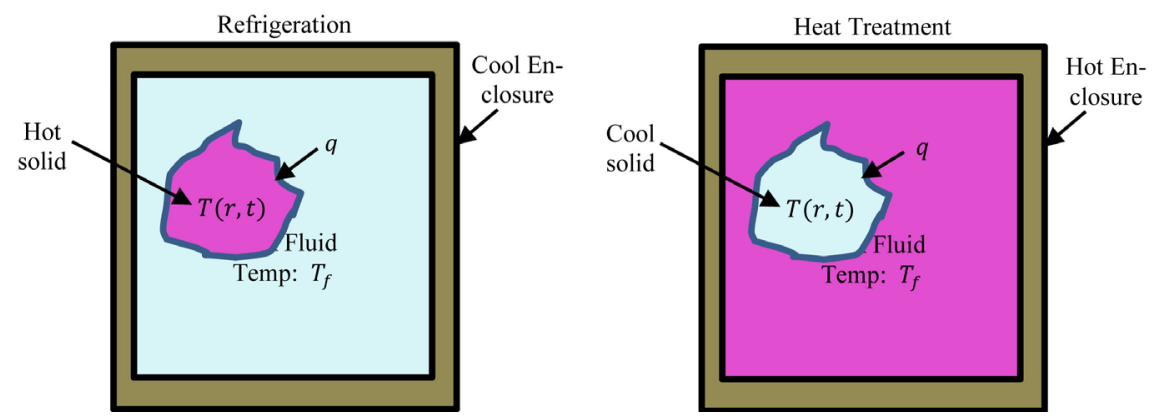

Figure 1. Heat transferring solid submerged in fluid. 

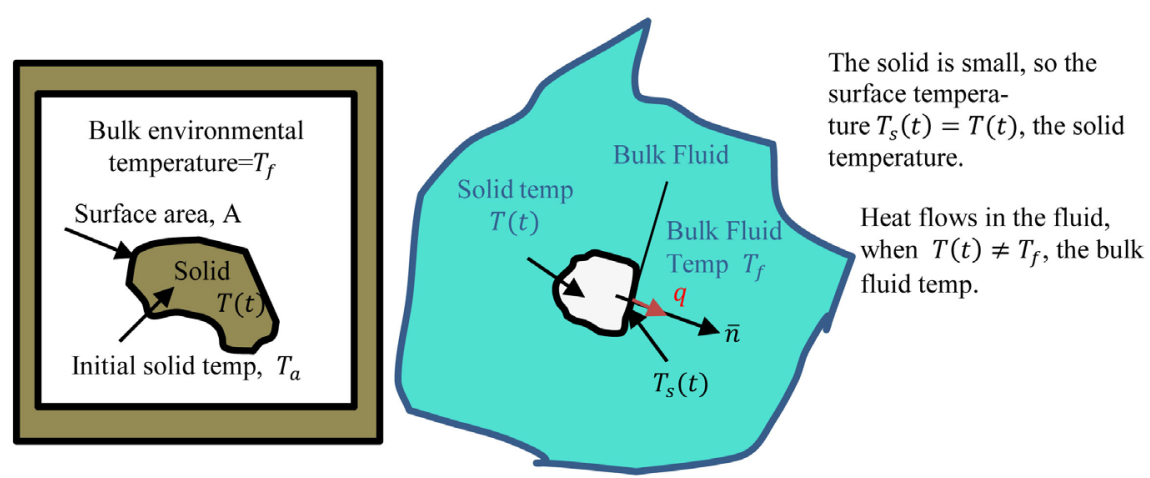

Figure 2. Mathematical model of heat transfer.

$$
q=h\left[T_{s}(t)-T_{f}\right]=h\left[T(t)-t_{f}\right] .
$$

In the above equation the coefficient $h$ satisfies the heat transformation between the solid and bulk fluid. Also the $1^{\text {st }}$ law of thermodynamic required that, to produce temperature change in a solid $\Delta T(t)$ during the time period $\Delta t$ can be obtain by the principle.

$$
-\rho c V \Delta T(t)=Q=q A_{s} \Delta t=h A_{s}\left[T(t)-T_{f}\right] \Delta t .
$$

From Equation (7), we have

$$
\frac{\Delta T(t)}{\Delta t}=-\frac{h}{\rho c V} A_{s}\left[T(t)-T_{f}\right] .
$$

In the above Equation (8) $h, \rho, c$ and $V$ on the right-hand-side are constant so we equating the ration of these constant with $\alpha$

$$
\alpha=\frac{h}{\rho c V} \text {. }
$$

Further Equation (8) can be expressed as

$$
\frac{\Delta T(t)}{\Delta t}=-\alpha A_{s}\left[T(t)-T_{f}\right] .
$$

Thus the change of the temperature of the submerged solid $T(t)$ is continuous with respect to time $t$, i.e. $\Delta t \rightarrow 0$ and if we replace the constant surface area as to a generic symbol A, we can express Equation (10) in the form of first-order differential equation [8]

$$
\frac{\mathrm{d} T(t)}{\mathrm{d} t}=-\alpha A_{s}\left[T(t)-T_{f}\right] .
$$

with initial condition

$$
\left.T(t)\right|_{t=0}=T(0)=T_{0}
$$

\section{Example 2.}

The initial temperature of an object is $80^{\circ} \mathrm{C}$ we place it in a refrigerator and maintained it in $5^{\circ} \mathrm{C}$. If $\alpha=0.002 / \mathrm{m}^{2} \cdot \mathrm{s}$ and $A=0.2 \mathrm{~m}^{2}$ find the time in which the object became cool down [8].

Solution. 
Here $t_{0}=80^{\circ} \mathrm{C}, T_{f}=5^{\circ} \mathrm{C}, \quad \alpha=0.002 / \mathrm{m}^{2} \cdot \mathrm{s}$ and $A=0.2 \mathrm{~m}^{2}$ using these values in Equation (11) we get

$$
\frac{\mathrm{d} T(t)}{\mathrm{d} t}=-(0.002)(0.2)[T(t)-5]=-0.0004[T(t)-5] .
$$

with the condition $T(0)=80^{\circ} \mathrm{C}$ then the above equation can be written as

$$
\frac{\mathrm{d} T(t)}{T(t)-5}=-0.0004 \mathrm{~d} t
$$

After integrating we get the solution.

$$
T(t)-5=\mathrm{e}^{-0.0004 t+c}=c \mathrm{e}^{-0.0004 t} .
$$

By using the initial condition we obtain the value of $c=75$ consequently the solution is

$$
T(t)=5+75 \mathrm{e}^{-0.0004 t} .
$$

If $t_{e}$ denote the time in which the temperature of the become down from $80^{\circ} \mathrm{C}$ to $8^{\circ} \mathrm{C}$, we get

$$
T\left(t_{e}\right)=8=5+75 \mathrm{e}^{-0.0004 t_{e}} .
$$

Solve Equation (13) for $t_{e}=8047 \mathrm{~s}$ or $2.24 \mathrm{~h}$.

\section{Example 3.}

The initial temperature of a turkey is $40^{\circ} \mathrm{F}$; if we put it into oven of a $350^{\circ} \mathrm{F}$ for an hour the temperature of the turkey become $100^{\circ} \mathrm{F}$. Find the temperature of the turkey after two hour and if the temperature of the turkey becomes $170^{\circ} \mathrm{F}$ haw many time will take it [7].

\section{Solution.}

The constant temperature of the oven is $t_{a}=350^{\circ} \mathrm{F}$ so from the solution of Equation (11) we get

$$
T(t)=c \mathrm{e}^{r t} .
$$

The initial temperature is $T(0)=40^{\circ} \mathrm{F}$, and this implies $40=350+C$ and hence $c=-310$.

To obtain the value of $r$, we put $T(1)=100$ in the last relation. So we have

$$
100=T(1)=350-310 \mathrm{e}^{r} .
$$

Solving the above equation for $r$ we get

$$
r=-0.21511 \text {. }
$$

The temperature of turkey after two hour is $T(2)=350-310 \mathrm{e}^{2 r} \approx 148.39^{\circ} \mathrm{F}$ for the second part we want to find $t$ so that $T(t)=170$, that is, solve $170=T(t)=350-310 \mathrm{e}^{r t}$. From the solution of this equation we obtain the required time $r t=\ln \frac{18}{31}$ so $t \approx 2 \mathrm{~h} 32 \mathrm{~min}$.

\section{Result}

Every physical phenomenon can be represented by ordinary differential equa- 
tion. A heat transferring in fluid can be represent by first-order differential equation, when the coefficient of this equation is constant then it called homogenous differential equation if the coefficient is not constant then the equation is non homogeneous differential equation. By the help of the first-order homogenous and non-homogeneous differential equation and by Newton law of cooling we can determine the problems of heat convection in fluid.

\section{Conclusion}

We have seen that the application of the first-order linear ordinary differential equation in heat problem is useful in mathematics and physics for instance in analyzing problems involving heat convection in fluid problem. This procedure is also used for calculating of heat transferring by conduction in solid and heat transferring by radiation in space. Beside the application of the first-order linear differential equation we divided these equations into two type homogeneous and non-homogeneous ordinary differential equation and solved these equations by the method of "separation variable". Also in this paper we find the optimal solution of the first-order homogeneous and non-homogeneous linear differential equation, which is very important in the solution of ordinary differential equation.

\section{Conflicts of Interest}

The author declares no conflict of interest regarding the publication of this paper.

\section{References}

[1] Keryszig, E. (2006) Advance Engineering Mathematics. 9th Edition, Willy, India. https://muquestionpapers.com/EngineeringBooks.php

[2] Caronongan, K. (2010) An Application of Differential Equations in the Study of Elastic Columns. Research Papers, Paper 5. http://opensiuc.lib.siu.edu/gs_rp/5

[3] Karthikeyan, N. and Jayaraja, A. (2016) Application of First Order Differential Equation to Heat Transfering in Solid. International journal of engineering and innovation technology, 5.

https://www.researchgate.net/publication/341575994_Application_of_First_Order_ diffe

[4] Michael, et al. (1995) Bay Delta Forum in Importance of Water Temperature in Aquatic System. Delta. http://cwemf.org/Pubs/BDMFTempReview.pdf

[5] Hassan, A. and Zakari, Y. (2018) Application of 1st Order Differential Equation in Temperature Problem. Annuls, Computer Science Series, 16th Tome 1st Fasc. https://anale-informatica.tibiscus.ro/download/lucrari/16-1-01-Hassan.pdf

[6] Kozhanov, A.I. (2014) First Russian-French Conference on Mathematical Geophysics, Mathematical Modeling in Continuum Mechanics and Inverse Problem. Magique 3D. https://team.inria.fr/magique3d/en/

[7] Adkins, W.A. and Davidson, M.G. (2010) Ordinary Differential Equation. Springer, USA. https://www.springer.com/gp/book/9781461436171

[8] Hsu, T.-R. (2005) Application of First Order Ordinary Differential Equation in Mechanical Engineering Analysis. San Jose State University, USA.

https://www.engr.sjsu.edu/trhsu/Chapter\%203\%20First\%20order\%20DEs.pdf 